\title{
Correction to: Preface of the Special Issue: International Symposium "Worlds of Entanglement" - Second Part
}

\author{
Diederik Aerts $^{1} \cdot$ Massimiliano Sassoli de Bianchi ${ }^{1,2} \cdot$ Sandro Sozzo $^{3} \cdot$ Tomas Veloz $^{1,4}$
}

Published online: 31 March 2021

(c) Springer Nature B.V. 2021

\section{Correction to: Foundations of Science https://doi.org/10.1007/s10699-021-09785-2}

In the original publication of the article, the sentence "Query ID="Q1" Text="Kindly check and confirm whether the corresponding author is correctly identified."' was inadvertently published in the first sentence of the first paragraph.

The sentence "This special issue is the second outcome of the International Symposium "Worlds of Entanglement," held at the Free University of Brussels (VUB), Query $\mathrm{ID}=$ "Q1" Text= "Kindly check and confirm whether the corresponding author is correctly identified." on September 29-30, 2017, which had a..." should read as "This special issue is the second outcome of the International Symposium "Worlds of Entanglement," held at the Free University of Brussels (VUB), on September 29-30, 2017, which had a...”.

The original article has been corrected.

Publisher's Note Springer Nature remains neutral with regard to jurisdictional claims in published maps and institutional affiliations.

The original article can be found online at https://doi.org/10.1007/s10699-021-09785-2.

Tomas Veloz

tveloz@gmail.com

Diederik Aerts

diraerts@vub.ac.be

Massimiliano Sassoli de Bianchi msassoli@vub.ac.be

Sandro Sozzo

s831@leicester.ac.uk

1 CLEA, Brussels Free University, Brussels, Belgium

2 Laboratorio di Autoricerca di Base, Lugano, Switzerland

3 School of Business and Centre IQSCS, University of Leicester, Leicester, UK

4 Fundación para el Desarrollo Interdisciplinario de la Ciencia, la Tecnología y las Artes, Santiago, Chile 